\title{
Energy levels and radiative rates for optically allowed and forbidden transitions of Ni XXV ion ${ }^{\star}$
}

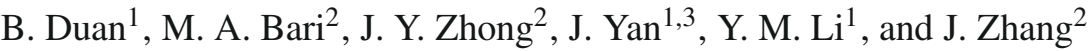 \\ 1 Institute of Applied Physics and Computational Mathematics, Beijing 100088, PR China \\ e-mail: duan_bin@iapcm.ac.cn \\ 2 Laboratory of Optical Physics, Institute of Physics, CAS, Beijing 100080, PR China \\ 3 Center for Applied Physics and Technology, Peking University, 100871, PR China
}

Received 3 March 2008 / Accepted 19 May 2008

ABSTRACT

\begin{abstract}
Aims. We report calculations of energy levels and radiative rates for 1300 fine-structure levels generated from 189 configurations of Be-like Nickel.

Methods. The General Purpose Relativistic Atomic Structure Program (GRASP2), developed by the I P Grant group in 1989 and partly improved by our group, is adopted in the calculations of energy levels and radiative rates.

Results. Our calculations are compared with those of other numerical methods and experiments so that their accuracy can be assessed. Additionally, the wavelengths, oscillator strengths, and radiative rates (for a given radiative rate and level structure, it is then easy to derive the line strength) are reported for all electric dipole (E1), electric quadrupole (E2), magnetic dipole (M1) and magnetic quadrupole (M2) transitions among these levels. Finally, the lifetimes of all excited levels are reported.
\end{abstract}

Key words. atomic data

\section{Introduction}

Radiative transition rates of highly ionized atoms have many important applications in determining the electron density and temperature of the solar corona and solar flares. These data are also required in studying the energy loss in controlled thermonuclear plasmas. A spectral line at $238.82 \AA$ was observed in a solar flare spectrum as described by Sandlin et al. (1976) who tentatively identified the line as the $2 \mathrm{~s}^{2}{ }^{1} \mathrm{~S}_{0} \leftarrow 2 \mathrm{~s} 2 \mathrm{p}{ }^{3} \mathrm{P}_{1}$ transition of Be-like $\mathrm{Ni}$ by the extrapolation along the isoelectronic sequence. The Be-like $\mathrm{Ni}$ lines are useful for measuring the temperature in flaring plasmas $(5 \sim 16) \times 10^{7} \mathrm{~K}$ as described by Landi et al. (2001). At temperatures (comparable to those of solar flares) in Tokamak plasmas, atoms such as chromium, iron, and nickel are important impurity elements and can be ionized to HeI-like of their ions as described by Goeler (1978). Therefore, atomic data (energy levels, radiative rates, collision strengths, and excitation rates) for Ni XXV are required for the spectroscopic observations of solar flares, laboratory produced plasmas, described by Fawcett et al. (1979), and as diagnostics for their physical parameters such as temperature and density. Moreover, Magnetic-dipole (M1) and Magnetic quadrupole (M2) transitions play a key role in high temperature low-density plasmas and are of great importance to plasma diagnostics, for instance, the $1 \mathrm{~s} 2 \mathrm{p}^{3} \mathrm{P}_{2} \rightarrow 1 \mathrm{~s}^{2}{ }^{1} \mathrm{~S}_{0}$ transition in He-like and Be-like ions as described by Beyer \& Shevelko (2003). Atomic transitions of the type $2 \mathrm{~s} 2 \mathrm{p}{ }^{3} \mathrm{P}_{2} \rightarrow 2 \mathrm{~s} 2 \mathrm{p}{ }^{3} \mathrm{P}_{1}$ in Be-like ions with $24 \leq Z \leq 30$ are expected to appear in the Solar Ultraviolet Measurements of Emitted Radiation (SUMER) wavelength range, described by Feldman et al. (2000). Among these chemical elements, only Fe

* Tables 1,3-6 are only available in electronic form at the CDS via anonymous ftp to cdsarc.u-strasbg. fr $(130.79 .128 .5)$ or via http://cdsweb.u-strasbg.fr/cgi-bin/qcat?J/A+A/488/1155 and Ni have sufficiently high solar abundance to justify the expectations that their lines might be visible in the flare spectra. The Be-like Fe line at $1079.415 \AA$ was detected presumably in the May 9th, 1999 solar flare but the plasma temperature was insufficiently high for a significant population of Be-like $\mathrm{Ni}$ ions to exist (2000). This line $2 \mathrm{~s} 2 \mathrm{p}{ }^{3} \mathrm{P}_{2} \rightarrow 2 \mathrm{~s} 2 \mathrm{p}{ }^{3} \mathrm{P}_{1}$ in Be-like Ni is both E2 and M1 transitions, with M1 being the largest component (see Tables 4 and 5), and atomic data for both E2 and $\mathrm{M} 1$ transitions of the $\mathrm{Be}$-like $\mathrm{Ni}$ are also required for solar flare plasma diagnostics.

Computations of the atomic parameters of $\mathrm{Ni}$ XXV ions have been attempted, in particular energy levels and radiative rates or oscillator strengths. Among the available data, the most notable one is provided by Fawcett $(1984,1985)$ by employing the Cowan's atomic structure codes described by Cowan (1967, 1968, 1981). Bhatia et al. (1986) calculated the radiative rates, oscillator strength, and the collision strength of the Be I-like isoelectronic sequence by using the energy levels of Ni XXV calculated by Edlén (1983) and Fawcett (1984). Zhang et al. (1992) used the fully relativistic methods and reported very few oscillator strengths (15 transitions) of the Be-like ions, such as the Ni XXV ion. These authors employed the multiconfiguration Dirac-Fock (MCDF) codes of Grant et al. (1980) and McKenzie et al. (1980) and improved by Dyall et al. (1989), which is an earlier version of the GRASP2 codes. Tully et al. (2004) calculated the radiative data for the 98 fine-structure levels among 45 configurations of Ni XXV by using Hibbert's configuration interaction codes CIV3 (Hibbert 1975; Hibbert et al. 1991), which includes semi-relativistic effects by means of the Breit-Pauli approximation. To construct wavefunctions, they employed configuration interaction (CI) among the levels, generated by the 45 configurations ( $\left.1 \mathrm{~s}^{2} 2 \ell n \ell^{\prime}, n=2-4\right)$. We note from Tully et al. (2004) that their comparison is limited to levels below the 47 th level due to the paucity of atomic data calculations, 
which are the calculations of the electric dipole (E1) transitions, and the order of the energy levels is found to differ from those of Fawcett et al. (1984). This reference indicated that the high lying states in Ni XXV are equally important, and it is necessary to extend the calculations into high lying excited levels. In plasma modelling, additionally, the other types of transitions, namely magnetic dipole (M1), electric quadrupole (E2), and magnetic quadrupole (M2), may also be required. And since Ni XXV is a moderately heavy ion $(Z=28)$, full relativistic effects may be of importance in the determination of wavefunctions and subsequently collision strength. Therefore, it is necessary to resolve the discrepancy between several energy levels (see Table 3 of Tully et al. 2004), and to extend both Tully et al. (2004) and Fawcett et al. (1984) calculations to higher lying excited levels, so that a system atomic data can be applied to the modelling of plasmas in the future.

The aim of our work is to perform a fully relativistic calculation and to derive the radiative data for all four types of transitions mentioned above by the improved GRASP 2 codes so as to assess the accuracy of the calculated and available data. Because there is no other theoretical and experimental energies for high lying excited states, we repeated our calculations with the Flexible Atomic Code (FAC) (Gu 2003). Both the GRASP2 and FAC codes solve the Dirac equation numerically, and include the two body relativistic operators. These operators are approximated as the Breit-Pauli approximation in the CIV3 calculations. In contrast to the LSJ coupling scheme adopted in the CIV3 calculations, these two codes are based on the JJ coupling scheme. The lifetimes for all excited levels of Ni XXV are listed with no comparison due to the lack of other theoretical or experimental results.

\section{Calculations procedure}

We took the important configuration interaction effect into account in the calculation of the structure of $\mathrm{Ni} \mathrm{XXV}$ ion with 189 configurations, which generate 1300 fine-structure levels. To calculate the atom structure with such a large number of basis states, the original GRASP2 codes published by Dyall et al. (1989) has been partly improved by our group. Firstly, among the four types of optimization modes for self-consistent calculation provided by the original GRASP2 codes, the extendedaverage-level (EAL) mode, which requires the input of level weights outside of the codes, is chosen to calculate the radial wave function, and the codes are developed to assign a weight automatically to each level. In our calculation, the weight is often defined by the fact that the high lying level always has a large weight. To ensure the convergence of the orbital functions, the selection order of the acceptable energy values are changed in the subroutine NEWE of the origin GRASP2 codes, which describes an energy adjustment procedure for finding an acceptable solution to the boundary value problem (Fischer 1986). Apart from the two above improvements, the calculations of the radial wave function are the same as that of EAL mode in the original GRASP2 codes as described by Dyall et al. (1989). Our numerical calculations indicate the weighted self-consistency of orbital functions, defined by Dyall et al. (1989), is lower less than $10^{-10}$. Secondly, since a large number of levels must be taken into account in our calculation, the dimensionality of matrix Hamiltonian of the atomic system is naturally large, and the calculations become extremely time-consuming if the Hamiltonian matrix is diagonalized using the methods provided by the original GPRASP2 codes. As completed by the GRASP92 codes (Parpia et al. 1996), the Hamiltonian matrix in the improved
Table 2. $N \operatorname{Ln\ell }(N l \leq n \ell)$ represents the following configurations.

\begin{tabular}{|c|c|c|c|}
\hline$N$ & $L$ & $n$ & $\ell$ \\
\hline 2 & 0,1 & $\begin{array}{c}6,7,8 \\
9\end{array}$ & $\begin{array}{c}0,1,2 \\
0,1\end{array}$ \\
\hline \multirow[b]{2}{*}{3} & 0,1 & $\begin{array}{c}5 \\
6,7,8 \\
9\end{array}$ & $\begin{array}{c}0,1,2,3 \\
0,1,2 \\
0,1\end{array}$ \\
\hline & 2 & $\begin{array}{c}5 \\
6,7 \\
8\end{array}$ & $\begin{array}{c}0,1,2,3 \\
0,1,2 \\
0,1\end{array}$ \\
\hline \multirow[t]{2}{*}{4} & 1 & $\begin{array}{c}5 \\
6 \\
7,8\end{array}$ & $\begin{array}{c}0,1,2,3 \\
0,1,2 \\
0,1\end{array}$ \\
\hline & 2,3 & $\begin{array}{c}5 \\
6,7\end{array}$ & $\begin{array}{c}0,1,2 \\
0,1\end{array}$ \\
\hline 5 & $0,1,2$ & $\begin{array}{c}5,6 \\
7\end{array}$ & $\begin{array}{c}0,1,2 \\
0,1\end{array}$ \\
\hline 6 & 0,1 & 6,7 & 0,1 \\
\hline 7 & 0,1 & 6,7 & 0,1 \\
\hline
\end{tabular}

codes are therefore rearranged into blocks, which each share the same total angular momentum and parity. Finally, to ensure that the calculations of the effective nuclear number $Z_{a}^{\text {eff }}$ in the selfenergy formula (see Eq. (56) of Dyall et al. 1989) successfully, some improvements were made in the subroutines QED (Dyall et al. 1989) of the original GRASP2 codes. Based on these improvements, the calculation of atomic structure, especially for those with a large number levels, were finished more easily and smoothly.

\section{Energy levels}

As mentioned before, the configurations $1 \mathrm{~s}^{2} 2 \ell n \ell^{\prime}(n=2-4)$ of the Ni XXV ion correspond to 98 fine-structure levels as listed in Table 1. Besides the these configurations, we also included the other 144 configurations ( $N L n \ell, N L \leq n \ell)$ in our calculations (see Table 2). All fine-structure levels generated by the above 189 configurations we calculated simultaneously by the improved GRASP2 and FAC codes. The calculated energies are presented in Table 1 along with those from the experimental compilations of NIST, which are available at the given website address in the reference section, and the calculated results of Tully et al. (2004). From Table 1, we can see that the numerical energies of the different methods are consistent with each other within a margin of the error of $0.6 \%$, and the difference between these results only become apparent in the fourth numerical figure of the energies with increasing the level energy. By comparing these three numerical results with those of NIST experiments, we found that they have almost same accuracy although the FAC and GRASP2 calculations are often slightly larger than the NIST values. Particularly, for the $2 \ell 2 \ell^{\prime}$ levels, the energies of CIV3 (2004) are in the best agreement with those of NIST, and both FAC and GRASP2 calculations appear to have similar poor accuracy. For the $2 \ell 3 \ell^{\prime}$ levels, the FAC calculations are consistently superior, with the GRASP2 calculations consistently the poorest, by the maximum amount of 0.27 Rydberg. When we compare our level energies with those of CIV3 (2004) and those from NIST (only 46 level energies are available from NIST data), we determined slightly different order of energy levels such as $31 / 32,65 / 66,73 / 74$, and $81 / 82$. But the order of energy levels by the FAC calculation are the same as for $73 / 74$, $81 / 82$, while an inverse order appears in the results of CIV3 calculations. For the levels: $13 / 14,31 / 32,65 / 66$, their orders are the 
same as in the results of FAC and CIV3, while they are in inverse order in the GRASP2 results. The reason why these three numerical results having extremely closed energies have different label order is that different numbers of configurations may produce slightly different level order. Finally, the lifetimes of these levels are listed in Table 1, as discussed in the following section.

\section{Wavelengths, oscillator strengths and radiative rates}

The radiative rates $A_{j i}$ (in $\mathrm{s}^{-1}$ units), i.e. transition probability, and absorption oscillator strength $\left(f_{i j}\right)$ for a transition $(j \rightarrow i)$ are related by the following expression as described by Tully et al. (2004):

$A_{j i}=\frac{8 \pi^{2} e^{2}}{m c \lambda_{j i}^{2}} \frac{g_{i}}{g_{j}} f_{i j}=\frac{6.671 \times 10^{15}}{\lambda_{j i}^{2}} \frac{g_{i}}{g_{j}} f_{i j}$

where $m$ and $e$ are the electron mass and charge, respectively, $c$ is the velocity of light, $\lambda_{j i}$ is the transition wavelength in $\AA$, and $g_{i}$ and $g_{j}$ are the statistical weights of the lower $i$ and upper $j$ levels, respectively. Some of the radiative rates of Ni XXV ion are calculated by the different codes (see Tully et al. 2004; Bhatia et al. 1986; NIST (NIST)). In this paper, all of the radiative rates are calculated by the improved GRASP2. From the results, we found that almost all transition probabilities (or oscillator strengths) of the strong lines have slight differences between the Coulomb and Babushkin gauge, so that for all allowed transitions (i.e. electric dipole (E1) transitions), only the values of both oscillator strengths $\left(g_{i} f_{i j}\right)$ and transition probabilities corresponding to Coulomb gauge $\left(A_{j i}\right)$ are listed (same for those of E2 transition in Table 4). The transition wavelengths $\left(\lambda_{j i}\right.$ in $\AA$ ) are also listed in Table 3. Given the oscillator strengths or transition probabilities, the line strengths are easily derived with the structure of energy levels in hand, so are not reported here. From Table 3, our calculated wavelengths are in close agreement with those reported by NIST (NIST), Tully et al. (2004), and Bhatia et al. (1986). For example, for the transition $4 \leftarrow 9$, its observed wavelength of NIST is $0.6 \%$ lower than $119.7,0.1 \%$ lower than Tully's result. Its transition probability of NIST is $4 \%$ lower than that shown in Table 3, but 5\% higher than the result of Tully et al. (2004). Similar results were found for the transition lines $1 \leftarrow 3$ and $1 \leftarrow 5$. Their observed wavelengths from NIST are 238.82 and $117.986 \AA$, respectively, while our results in Table 3 are 238.13 and $116.914 \AA$, respectively. For the transition line $1 \leftarrow 15$, the oscillator strength $\left(f_{i j}=0.4225\right)$ in Table 3 is very closed to the value 0.422 calculated by Bhatia et al. (1986), while it is 0.4290 in the case of CIV3 calculated by Tully et al. (2004). In Table 3, most of the transition probabilities are consistent with those of Tully et al. (2004), although some differences exist for a few lines. As an example, for the transition line, $1 \leftarrow 50$, our result is $23 \%$ higher than that of Tully et al. (2004).

For plasma diagnostic purposes in solar flares, a complete set of atomic data, i.e. electric quadrupole (E2), magnetic dipole (M1) and magnetic quadrupole (M2) transitions between all calculated levels, are calculated using the improved GRASP2 codes and reported in Tables 4-6.

By analyzing and evaluating these radiative results for the electric multipoles E1 and E2, we found that almost all strong lines between levels below 300th have slightly different oscillator strengths (or transition probabilities) corresponding to Coulomb and Babushkin gauge, and that only a few have different oscillator strengths (the difference between the oscillator strengths corresponding to Coulomb and Babushkin gauge is within about 20\%), which appears to indicate their calculation error should be lower than $20 \%$. Finally, we note that all these calculations have already been considered by the higher order effects such as Briet interaction and QED effect.

\section{Lifetimes}

The lifetime $\tau_{j}$ for a level $j$ are written as follows:

$\tau_{j}=\frac{1}{\sum_{i} A_{j i}}$.

This parameter can be measured empirically and provides a check of the accuracy of the numerical calculations. To our knowledge, no alternative experimental measurements of the lifetimes for the levels of Ni XXV exist. Table 1 presents our calculated lifetimes, which include contributions from four types of transitions i.e. E1, E2, M1, and M2. These results may be helpful for future comparison with theory calculations or experiments.

\section{Conclusions}

In this paper, we have presented the calculation of electric dipole (E1), electric quadrupole (E2), magnetic dipole (M1) and magnetic quadrupole (M2) transition wavelengths and transition probabilities for transition between the $n=2-4$ states of $\mathrm{Ni}$ XXV ion. To assess the accuracy of our results, two independent codes, i.e. the improved GRASP 2 and FAC codes, are used in our calculations. These reported results are in good agreement with both measurement and the latest numerical calculations, where comparison is possible.

Acknowledgements. This work is jointly supported by the NSFC under Grant Nos. 10675025, 10674020, 10674021, 60621063 and Natural Basic Research Program of China (973 program, Grant No. 2007CB815102).

\section{References}

Beyer, H. F., \& Shevelko, V. P. 2003, Introduction to the physics of highly charged ions (Bristol and Philadelphia: IOP publishing)

Bhatia, A. K., Feldman, U., \& Seely, J. F. 1986, At. Data Nucl. Data Tables, 35, 449

Cowan, R. D. 1967, Phys. Rev., 163, 54

Cowan, R. D. 1968, J. Opt. Soc. Am., 58, 808

Cowan, R. D. 1981, The Theory of Atomic Structure and Spectra (Los Angeles: University of California Press)

Dyall, K. G., Grant, I. P., Johnson, C. T., \& Plummer, E. P. 1989, Comput. Phys. Commun., 55, 425

Edlén, B. 1983, Phys. Scr., 28, 51

Fawcett, B. C. 1984, At. Data Nucl. Data Tables, 30, 1

Fawcett, B. C. 1985, At. Data Nucl. Data Tables, 33, 479

Fawcett, B. C., Ridgeley, A., \& Hughes, T. 1979, MNRAS, 188, 365

Feldman, U., Curdt, W., Landi, E., \& Wilhelm, K. 2000, ApJ, 544, 508

Fischer, C. F. 1986, Comput. Phys. Rep., 3, 273

Grant, I. P., McKenzie, B. J., Norrington, P. H., Mayers, D. F., \& Pyper, N. C. 1980, Comput. Phys. Commun., 21, 207

Goeler, S. V. 1978, Soft X-ray measurement, in Diagnostics for fusion experiments, Varenna, Italy (Oxford: Pergamon)

Gu, M. F. 2003, ApJ, 582, 1241

Hibbert, A. 1975, Comput. Phys. Commun., 9, 141

Hibbert, A., Glass, R., \& Froese Fischer, C. 1991, Comput. Phys. Commun., 64, 455

Landi, E., Doron, R., Feldman, U., \& Doschek, G. A. 2001, ApJ, 556, 912

McKenzie, B. J., Grant, I. P., \& Norrington, P. H. 1980, Comput. Phys. Commun., 21, 233

Parpia, F. A., Fischer, C. F., \& Grant, I. P. 1996, Comput. Phys. Commun., 94, 249

Sandlin, G. D., Brueckner, G. E., Scherrer, V. E., \& Tousey, R. 1976, ApJ, 205, L47

Tully, J. A., \& Chidichimo, C. M. 2004, J. Phys. B, 37, 689

Zhang, H. L., \& Sampson, D. H. 1992, At. Data Nucl. Data Tables, 52, 143

http://physics.nist.gov/asd3 